\title{
NLRP12-associated hereditary periodic fever syndrome
}

INSERM

\section{Source}

INSERM. (1999). Orphanet: an online rare disease and orphan drug data base. NLRP12associated hereditary periodic fever syndrome. ORPHA:247868

NLRP12-associated hereditary periodic fever syndrome is a rare autoinflammatory syndrome characterized by episodic and recurrent periods of fever combined with various systemic manifestations such as myalgia, arthralgia, joint swelling, urticaria, headache and skin rash. Common trigger of these episodes is cold. 